\title{
A Broadly Accessible Liquid Chromatography Method for Quantification of Six Nitrosamine Compounds and N,N-Dimethylformamide in Metformin Drug Products Using High Resolution Mass Spectrometry
}

\author{
Qian Wu, Evgenia Kvitko, Nicola Zenzola, Kaury Kucera,* David Y. Light \\ Valisure, New Haven, CT \\ *Corresponding author email: kucera@ valisure.com
}

\begin{abstract}
$\mathrm{N}$-nitrosodimethylamine (NDMA) and the industrial solvent, N,N-dimethylformamide (DMF), are both probable human carcinogens that have been detected in pharmaceutical drug products like metformin, which is used to treat type II diabetes. Some lots of metformin drug products have exceeded the United States Food and Drug Administration (FDA) daily allowable intake limit for NDMA, while the presence of DMF has been detected at several orders of magnitude higher than NDMA. A recent study found that a low abundance isotope of DMF interferes with NDMA quantification by using a unique subset of LC-MS instruments capable of high mass resolution. In this study, an LC-HRMS method is developed that chromatographically separates NDMA from DMF in metformin drug products to eliminate interference. The method can detect nitrosamines and DMF under the current regulatory guidance for industry and provides a solution for simultaneously quantifying nitrosamines and DMF for a broad range of LC-MS instruments.
\end{abstract}

\section{Keywords}

Nitrosamine, NDMA, DMF, Metformin, LC-MS, LC-HRMS

\section{Introduction}

A class of probable human carcinogens, nitrosamines, have been found in some drug products including angiotensin II receptor blockers (ARBs) valsartan and losartan, ranitidine, nizatidine, and metformin resulting in global recalls that have significantly disrupted pharmaceutical supply and affected healthcare industries beginning in 2018. ${ }^{1-3}$ Nitrosamines are a class of compounds with the chemical structure of a nitroso group bonded to an amine and are widely associated with carcinogenesis. ${ }^{4} \mathrm{~N}$-nitrosodimethylamine (NDMA) has been the most commonly detected nitrosamine in drug products and is also found in some food and drinks. ${ }^{5}$ To date, the five nitrosamines NDMA, N-nitrosodiethylamine (NDEA), N-nitroso-N-methyl-4-aminobutanoic acid (NMBA), N-nitrosoisopropylethyl amine (NEIPA), and N-nitrosomethylphenylamine (NMPA) have been reported in drug substances or products. Some other nitrosamines, namely 
N-nitrosodiisopropylamine (NDIPA) and N-nitrosodibutylamine (NDBA), may be theoretically present in drug products. ${ }^{6}$

Independent analysis of finished pharmaceutical drug products performed by private laboratories and academia has played an increasingly significant role in confirming potentially toxic contaminations in drug products and alerting both the public and regulatory agencies. ${ }^{7-10} \mathrm{~N}, \mathrm{~N}-$ dimethylformamide (DMF) may be used in some pharmaceutical manufacturing processes and, because nitrosation of DMF results in NDMA, industrial use has been proposed as the root cause of some NDMA contamination. ${ }^{6}$ DMF is itself considered to be a probable human carcinogen making its detection in valsartan and metformin drug products of great concern. ${ }^{11,12}$

Several nitrosamines and DMF have generated evidence of genotoxicity in animal studies and are therefore classified by the International Agency for Research on Cancer (IARC) of the World Health Organization (WHO) as "Group 2A" or "probable human carcinogens". ${ }^{13}$ The United States Food and Drug Administration (FDA) determines the daily allowable intake limit of contaminants in pharmaceuticals by approximating a 1:100,000 cancer risk after 70 years of exposure. ${ }^{14}$ The current limits are 96 nanograms per day for NDMA and NMBA, $26.5 \mathrm{ng}$ /day for NDEA, NMPA, NEIPA, and NDIPA, and 8.8 milligrams per day for DMF. ${ }^{6,15}$ International regulators have signaled the intent to further reduce the threshold limit of daily allowable intake of NDMA to potentially 0.03 parts per million ( $\mathrm{ppm}$ ) or less, ${ }^{16}$ making it increasingly imperative for industry to not only possess highly accurate and sensitive analytical methods but for these methods to be applicable to a wide range of laboratory instruments, as many analytical resources will be needed to test drug products. Authors have petitioned FDA to evaluate the nearly one hundred thousand-fold higher DMF permitted exposure and restrict its use in the pharmaceutical industry in light of its recent re-classification as "Group 2A" in 2018 and potential reactivity to form NDMA in the manufacturing process.

In March 2020, a citizen petition was filed to the FDA by Valisure to report detection of NDMA in excess of FDA limits for 16 of 38 tested metformin drug product lots. The analytical method used by the petitioner in the citizen petition was a liquid chromatography-high resolution mass spectrometry (LC-HRMS) method modified from a method published by the FDA. ${ }^{17}$ As a confirmation process, the FDA requested all 38 lots of metformin drug products from the petitioner and evaluated the method used in the citizen petition. In FDA's evaluation of the same lots of metformin samples provided by Valisure, extremely high mass resolution, which is only achievable by orbitrap mass spectrometers, was shown to be critical for accurate quantification of NDMA when DMF is also present. This is due to co-elution of NDMA and a rare isotope of DMF with a similar mass to NDMA. Specifically, the ${ }^{15} \mathrm{~N}$-DMF isotope (74.0493 Da) interfered with the detection of NDMA (74.0475 Da). Although removal of this interference reduced total NDMA detection in some samples, the FDA still determined that samples from 8 of the 16 suspected lots contained unacceptable levels of NDMA. ${ }^{18}$ To date, metformin drug products are still being recalled due to the NDMA contamination. The contamination of metformin drug products with DMF remains concerning and widespread efforts to detect and quantify DMF are warranted. 
The aim of the present study is to develop an LC-MS method that chromatographically separates NDMA and DMF so that nitrosamines and DMF can be analyzed simultaneously using a broad range of high-resolution mass spectrometry instruments. To our knowledge, this is also the first effort to develop analytical method that quantifies nitrosamines and DMF in pharmaceutical samples simultaneously.

\section{Materials and Methods}

\section{Chemicals and Reagents}

Six nitrosamines and DMF were selected as target analytes in the present study. Reference standards of NDMA, NDEA, NDBA, NEIPA, DMF, and isotope labeled DMF standard D7-DMF were purchased from Sigma-Aldrich (St. Louis, MO). Reference standards of NMBA and NDIPA were purchased from BOC Sciences (Shirley, NY). Isotopically labeled NDMA standard ${ }^{13} \mathrm{C}_{2}$ - $\mathrm{D}_{6}$-NDMA was purchased from Cambridge Isotope Laboratories (Tewksbury, MA). Formic acid (LC-MS grade) was purchased from Millipore Sigma (Burlington, MA). Methanol (LC-MS grade) was purchased from Honeywell (Charlotte, NC). Milli-Q water (18.2 M 2 ) was generated from a Milli-Q IQ Element system from Millipore Sigma.

\section{Sample Preparation}

A single metformin or metformin combination drug tablet is transferred to a $15-\mathrm{mL}$ polypropylene (PP) tube and accurately weighed. Methanol is added to the $15-\mathrm{mL}$ PP tube to dilute the tablet sample to approximately $100 \mathrm{mg}$ active pharmaceutical ingredient (API) per $\mathrm{mL}$ of methanol, and the volume of methanol is accurately recorded. The tablet sample in methanol is blended with a Pro2000 blender by Pro Scientific (Oxford, CT). Between samples, the probe of the blender is rinsed by performing blending action in a series of two 50-mL PP tubes with Milli-Q water and two 50-mL PP tubes with methanol. At the end of sample preparation of each batch, the last tube of rinsing methanol is analyzed along with the metformin samples as the laboratory reagent blank (LRB). The sample methanol extract is incubated at room temperature for 30 minutes prior to centrifugation at 13,000 rpm for 2 minutes. $250 \mu \mathrm{L}$ of the supernatant is transferred and diluted with $750 \mu \mathrm{L}$ of HPLC grade water (Sigma Aldrich), followed by vortex mixing. After filtering by $0.2 \mu \mathrm{m}$ nylon filter, $480 \mu \mathrm{L}$ of the sample extract is pipette-transferred to an amber $\mathrm{LC}$ vial, and $20 \mu \mathrm{L}$ of isotopic internal standard mixture containing $20 \mathrm{ng}$ of ${ }^{13} \mathrm{C}_{2}-$ $\mathrm{D}_{6}$-NDMA and $500 \mathrm{ng}$ of $\mathrm{D}_{7}$-DMF is spiked into the sample extract. The final sample extract contains $40 \mathrm{ng} / \mathrm{mL}^{13} \mathrm{C}_{2}-\mathrm{D}_{6}$-NDMA and $1 \mu \mathrm{g} / \mathrm{mL} \mathrm{D}_{7}$-DMF, respectively. The vial is capped and vortexed prior to LC-HRMS analysis.

\section{Instrumental Analysis}

SCIEX ExionLC AD (LC) coupled with SCIEX X500R quadrupole time-of-flight high resolution mass spectrometry (QToF HRMS) was purchased from SCIEX (Framingham, MA). Ten (10) $\mu \mathrm{L}$ of sample extract is injected and the separation of six nitrosamine analytes and DMF is performed on an InfinityLab Poroshell 120 EC-C18, $4.6 \times 100 \mathrm{~mm}, 2.7 \mu \mathrm{m}$ analytical column (Agilent Technology, Santa Clara, CA). LC mobile phase is $0.1 \%(\mathrm{v} / \mathrm{v})$ formic acid in 
water (A) and $0.1 \%(\mathrm{v} / \mathrm{v})$ formic acid in methanol (B). The remaining LC parameters are listed in Table 1.

Determination of target analytes is performed by SCIEX X500R QToF HRMS operated in positive atmospheric pressure ionization (APCI+) mode. Mass resolution for TOF MS scan was greater than 34,000 for amino-dPEG 4-acid calibrant mass of 266.15981 Da, and mass resolution for TOF MS/MS scan was greater than 31,000 for mass 176.09134 Da fragment of reserpine calibrant. The mass spectrometry method was high resolution multiple reaction monitoring (MRMHR), which has similar action compared to triple quadrupole mass spectrometry that allows the HRMS to detect precursor and product ions of target molecules, but with extremely high mass resolution detection. Exact masses for MRMHR mass transitions and other mass spectrometry parameters are listed in Table 2. Mass accuracy for quantification is set at $5 \mathrm{ppm}$ for all analytes. The total run time is 15 minutes for each sample. Metformin API is diverted to waste in the first 4 minutes of the LC gradient.

\section{Quality Assurance and Quality Control (QA \& QC)}

All calibration and QC samples are prepared in 25/75 (v/v) methanol/water. A nine-point standard calibration ranged from $0.25 \mathrm{ng} / \mathrm{mL}$ to $80 \mathrm{ng} / \mathrm{mL}$ for six nitrosamines, and $25 \mathrm{ng} / \mathrm{mL}$ to $8000 \mathrm{ng} / \mathrm{mL}$ for DMF is analyzed on LC-QToF HRMS. Isotopic dilution calibration method is used for NDMA and DMF quantification with quadratic regression and 1/x weighting, while external calibration method is used for the rest of the analytes with linear regression and $1 / \mathrm{x}$ weighting, and $\mathrm{R}^{2}$ for all calibrations need to be greater than 0.99 . A calibration standard is acceptable if the calculated concentration is within $\pm 20 \%$ of its theoretical concentration, except for the lowest acceptable concentration which is $\pm 30 \%$. Limit of quantification (LOQ) is acceptable for the lowest calibration standard that meets the following three criteria 1) signal to noise ratio is not less than $10 ; 2$ ) the calculated concentration is within $\pm 30 \%$ of its theoretical concentration; and 3) the calculated concentration is not less than three times higher than the concentration in the laboratory reagent blank (LRB) if the LRB has a detectable concentration. Low, medium, and high QC samples are prepared following the same procedure of sample preparation, and a QC is acceptable if the calculated concentration is within $\pm 20 \%$ of its theoretical concentration.

\section{GC-MS Analysis for Validation of DMF Analyzed by LC-MS}

GC-MS methodology is commonly used for the detection of residual solvent including DMF in pharmaceutical drug products. Five lots of metformin drug product samples obtained from Valisure Pharmacy were analyzed following USP <467> Residual Solvents Method and the LCMS method developed in the present study in parallel. Analysis of each lot of metformin samples were performed in triplicate to evaluate the repeatability of the method.

\section{Results and Discussion}

\section{LC Separation of Six Nitrosamines and DMF}


Retention times for target analytes are $4.11 \mathrm{~min}$ for ${ }^{13} \mathrm{C}_{2}-\mathrm{D}_{6}$-NDMA, $4.22 \mathrm{~min}$ for NDMA, 4.35 min for $\mathrm{D}_{7}$-DMF, $4.53 \mathrm{~min}$ for DMF, $7.09 \mathrm{~min}$ for NMBA, $8.20 \mathrm{~min}$ for NDEA, $8.93 \mathrm{~min}$ for NEIPA, $9.48 \mathrm{~min}$ for NDIPA, and $10.63 \mathrm{~min}$ for NDBA. An example of separation of NDMA and DMF is shown in Figure 1, presenting a $10 \mathrm{ng} / \mathrm{mL}$ nitrosamine and $1000 \mathrm{ng} / \mathrm{mL}$ DMF analytical standard mixture. This result suggests that when high concentration of DMF presents in a sample, at normal QToF instrument mass resolution (about 30,000 near mass 200) and with 5 ppm mass accuracy, ${ }^{15} \mathrm{~N}-\mathrm{DMF}$ (75.0571), which only constitutes approximately $0.4 \%$ of native DMF abundance, could interfere with the mass detection of NDMA (75.0553). Figure 2 shows the detection of native DMF (74.0600) in the same standard used in Figure 1. The chromatographic method in the present study separates NDMA and DMF by 0.3 minute and has made the quantification of both compounds possible. Figure 3 shows the detection of NDMA in a real metformin extended release drug sample without the interference of DMF.

\section{Limit of Quantification}

The limit of quantification for each target compound is determined by the criteria described above. Continuous monitoring of LRBs does not show detectable concentration of target compounds in the blanks. The LOQs are $0.25 \mathrm{ng} / \mathrm{mL}$ for NDMA, $25 \mathrm{ng} / \mathrm{mL}$ for DMF, $1 \mathrm{ng} / \mathrm{mL}$ for NMBA, $0.25 \mathrm{ng} / \mathrm{mL}$ for NDEA, $0.25 \mathrm{ng} / \mathrm{mL}$ for NEIPA, $0.25 \mathrm{ng} / \mathrm{mL}$ for NDIPA, and 0.25 $\mathrm{ng} / \mathrm{mL}$ for NDBA. The reporting limits are sample-dependent and are calculated based on the dilution factor used in the sample preparation for each sample. For example, in a metformin drug tablet of $500 \mathrm{mg}$ dosage, the LOQ for NDMA is approximately $5 \mathrm{ng} /$ tablet and $0.01 \mathrm{ppm}$ API following the sample preparation procedure described above. Table 3 shows the calculated nitrosamine dose per day if they are detected at LOQ concentrations, and a patient takes up to $2000 \mathrm{mg}$ of metformin as maximum dosage prescription. $2000 \mathrm{mg}$ per day is the recommended maximum for extended release formulations while immediate release formulations can be prescribed up to $2550 \mathrm{mg}$ per day. The LOQ equivalent dosages are below the allowable intake in the guidance for industry issued by the FDA in September $2020 .^{6}$

\section{Accuracy and Precision}

Calibration curves for each of the seven target compounds demonstrated accuracies within 20\% of the expected concentrations at each calibration level. Continuous monitoring of low $(1 \mathrm{ng} / \mathrm{mL}$ nitrosamines and $100 \mathrm{ng} / \mathrm{mL}$ DMF), medium ( $5 \mathrm{ng} / \mathrm{mL}$ nitrosamines and $500 \mathrm{ng} / \mathrm{mL}$ DMF), and high (20 ng/mL nitrosamines and $2000 \mathrm{ng} / \mathrm{mL}$ DMF) levels shows less than $15 \%$ RSD for all analytes. Figure 4 shows NDMA and DMF calibration curves generated for the present study.

\section{Validation of DMF Analysis by GC-MS}

Table 4 shows a summary of result comparison of the analysis of five lots of metformin drug product samples by USP <467> GC-MS and LC-MS method. The results obtained from the two methods show a good agreement with less than $30 \%$ difference in DMF detection between the two methods. For the LC-MS method, the relative standard deviation is, on average, lower than that of GC-MS method and is less than $10 \%$ for each of the five samples analyzed. NDEA, NDBA, NMBA, NEIPA, and NDIPA are not detected in any of the metformin samples. 


\section{Conclusion}

An LC-QToF HRMS method is developed and validated for the analysis of NDMA, DMF, and five other nitrosamines in metformin drug products after the target analytes are separated by Agilent InfinityLab Poroshell $120 \mathrm{EC}-\mathrm{C} 18,4.6 \times 100 \mathrm{~mm}, 2.7 \mu \mathrm{m}$ analytical column. The sensitivity of the method is sufficient that the LOQ equivalent of daily nitrosamine exposure is lower than FDA's guidance to industry. The method enables a QToF high resolution mass spectrometry or other types of mass spectrometry to perform precision analysis of NDMA and DMF simultaneously for samples where both these probable human carcinogens are present at concerningly high levels. Though this method is validated for metformin drug products, it can be optimized and validated for other pharmaceutical drug products. 


\section{References}

1. U.S. FDA. Recalls of Angiotensin II Receptor Blockers (ARBs) including Valsartan, Losartan and Irbesartan. www.fda.gov/drugs/drug-safety-and-availability/recalls-angiotensin-ii-receptorblockers-arbs-including-valsartan-losartan-and-irbesartan. Published 2020. Accessed.

2. U.S. FDA. FDA Updates and Press Announcements on NDMA in Zantac (ranitidine). www.fda.gov/drugs/drug-safety-and-availability/fda-updates-and-press-announcements-ndmazantac-ranitidine. Published 2020. Updated 16 April 2020. Accessed.

3. U.S. FDA. FDA Updates and Press Announcements on NDMA in Metformin. www.fda.gov/drugs/drug-safety-and-availability/fda-updates-and-press-announcements-ndmametformin. Published 2020. Updated 5 October 2020. Accessed.

4. Robles H. In. Encyclopedia of Toxicology (Third Edition): ScienceDirect; 2014:Pages 584-585.

5. Gushgari AJ, Halden RU. Critical review of major sources of human exposure to N-nitrosamines. Chemosphere. 2018;210:1124-1136.

6. (CDER) FaDACfDEaR. Control of Nitrosamine Impurities in Human Drugs Guidance for Industry. In: Services USDoHaH, ed2020.

7. Valisure Citizen Petition on Ranitidine. 2019. www.regulations.gov/document?D=FDA-2019-P4281-0001.

8. Valisure Citizen Petition on Metformin. 2020. beta.regulations.gov/document/FDA-2020-P-09780001.

9. Request that the FDA suspend sale of all lots of all products containing ranitidine. 2020. beta.regulations.gov/docket/FDA-2020-P-0042.

10. Citizen Petition from University of Kentucky Drug Quality Study. 2020. beta.regulations.gov/document/FDA-2020-P-2033-0001.

11. Valisure Citizen Petition on N,N-Dimethylformamide. 2019. beta.regulations.gov/docket/FDA2019-P-2869.

12. Valisure. Comment from Valisure LLC RE: Comment on Citizen Petition on N,NDimethylformamide. In. Regulations.gov2020.

13. List of Classifications. World Health Organization; 2020. monographs.iarc.fr/list-ofclassifications.

14. U.S. Department of Health and Human Services Food and Drug Administration CfDEaRC, Center for Biologics Evaluation and Research (CBER). M7(R1) Assessment and Control of DNA Reactive (Mutagenic) Impurities in Pharmaceuticals To Limit Potential Carcinogenic Risk Guidance for Industry. 2018.

15. Center for Drug Evaluation and Research (CDER) CfBEaRC. Q3C - Tables and List Guidance for Industry. In: Services USDoHaH, ed: U.S. Food \& Drug Administration; 2018.

16. (CHMP) CfMPfHU. Assessment report, Referral under Article 31 of Directive 2001/83/EC angiotensin-II-receptor antagonists (sartans) containing a tetrazole group. In: European Medicines Agency 2019.

17. U.S. FDA. Liquid Chromatography-High Resolution Mass Spectrometry (LC-HRMS) Method for the Determination of NDMA in Metformin Drug Substance and Drug Product. 4 February 2020 2020. FY20-058-DPA-S.

18. Yang J, Marzan TA, Ye W, Sommers CD, Rodriguez JD, Keire DA. A Cautionary Tale: Quantitative LC-HRMS Analytical Procedures for the Analysis of N-Nitrosodimethylamine in Metformin. AAPS J. 2020;22(4):89. 
Table 1. LC parameters used for separating six nitrosamines and DMF in the present study.

\begin{tabular}{|c|c|c|c|}
\hline Column Temp. & \multicolumn{3}{|l|}{$30^{\circ} \mathrm{C}$} \\
\hline Flow Rate & \multicolumn{3}{|l|}{$0.6 \mathrm{~mL} / \mathrm{min}$} \\
\hline \multirow[t]{7}{*}{ Gradient } & Time (min) & Flow Rate (mL/min) & B $\%$ \\
\hline & 0 & 0.6 & 2 \\
\hline & 3.5 & 0.6 & 2 \\
\hline & 9.5 & 0.6 & 98 \\
\hline & 10.9 & 0.6 & 98 \\
\hline & 11 & 0.6 & 2 \\
\hline & 15 & 0.6 & 2 \\
\hline Autosampler Temperature & \multicolumn{3}{|l|}{$4^{\circ} \mathrm{C}$} \\
\hline Needle Wash & \multicolumn{3}{|c|}{$\begin{array}{l}\text { Methanol: Water }(80: 20) \text { with } 0.1 \% \text { Formic } \\
\text { Acid }\end{array}$} \\
\hline
\end{tabular}

Table 2. SCIEX X500R QToF HRMS parameters for determination of six nitrosamines and $\mathrm{DMF}$ in the present study.

\begin{tabular}{|c|c|c|c|}
\hline \multicolumn{4}{|l|}{ Source and Gas Parameters } \\
\hline Ion source gas 1 & 45 psi & Curtain gas & $25 \mathrm{psi}$ \\
\hline Temperature & $325^{\circ} \mathrm{C}$ & CAD gas & 8 \\
\hline \multicolumn{4}{|l|}{ Experiment Parameters } \\
\hline Polarity & Positive & Nebulizer current & $3 \mu \mathrm{A}$ \\
\hline \multicolumn{2}{|l|}{ TOF MS } & Accumulation time & $0.1 \mathrm{~s}$ \\
\hline TOF start mass & $50 \mathrm{Da}$ & TOF stop mass & $450 \mathrm{Da}$ \\
\hline Declusting potential/spread & $40 / 0 \mathrm{~V}$ & $\begin{array}{l}\text { Collision } \\
\text { energy/spread }\end{array}$ & $10 / 0 \mathrm{~V}$ \\
\hline \multicolumn{2}{|l|}{ TOF MS/MS } & Accumulation time & $0.025 \mathrm{~s}$ each \\
\hline Compound & Precursor (Da) & Product (Da) & DP/CE (V) \\
\hline NDMA & 75.06 & 75.0553 & $80 / 5$ \\
\hline NDMA(Q) & 75.06 & 43.0291 & $80 / 22$ \\
\hline${ }^{13} \mathrm{C}_{2} \mathrm{D}_{6}$-NDMA & 83.10 & 83.0997 & $80 / 5$ \\
\hline${ }^{13} \mathrm{C}_{2} \mathrm{D}_{6}-\mathrm{NDMA}(\mathrm{Q})$ & 83.10 & 47.0513 & $80 / 22$ \\
\hline \multirow[t]{3}{*}{ NMBA } & 147.08 & 147.0764 & $40 / 5$ \\
\hline & 147.08 & 117.0784 & $40 / 8$ \\
\hline & 147.08 & 44.0493 & $40 / 15$ \\
\hline
\end{tabular}




\begin{tabular}{|l|l|l|l|}
\hline \multirow{2}{*}{ NDEA } & 103.09 & 75.0553 & $40 / 15$ \\
\cline { 2 - 4 } & 103.09 & 103.0866 & $40 / 15$ \\
\hline NEIPA & 117.10 & 75.0553 & $40 / 20$ \\
\hline NDIPA & 131.12 & 131.1179 & $40 / 5$ \\
\hline NDBA & 159.15 & 57.0704 & $40 / 25$ \\
\cline { 2 - 4 } & 159.15 & 103.0872 & $40 / 15$ \\
\cline { 2 - 4 } & 159.15 & 159.1492 & $40 / 15$ \\
\hline DMF & 74.06 & 74.0600 & $100 / 5$ \\
\hline D $_{7}$-DMF & 81.10 & 81.1040 & $100 / 5$ \\
\hline
\end{tabular}

Table 3. The LOQ equivalent of nitrosamine dose per day calculated for one takes the common maximum daily dose of $2000 \mathrm{mg}$ metformin.

\begin{tabular}{|l|l|l|}
\hline Compound & $\begin{array}{l}\text { AI Limit } \\
\text { (ng/day) }\end{array}$ & $\begin{array}{l}\text { LOQ Equivalent for 2000 mg API/day } \\
\text { (ng/day) }\end{array}$ \\
\hline NDMA & 96 & 20 \\
\hline NMBA & 96 & 80 \\
\hline NDEA & 26.5 & 20 \\
\hline NEIPA & 26.5 & 20 \\
\hline NDIPA & 26.5 & 20 \\
\hline
\end{tabular}

Table 4. Result summary of DMF analysis of five lots of metformin drug products by LC-MS and GC-MS method ( $\mathrm{n}=3$ for each analysis).

\begin{tabular}{|c|c|c|c|c|c|c|c|c|c|}
\hline \multirow{2}{*}{ Sample } & \multirow{2}{*}{ Type } & \multirow{2}{*}{ Dosage (mg) } & \multicolumn{3}{|c|}{ LCMS } & \multicolumn{3}{|c|}{ GCMS (USP 467) } & \multirow{2}{*}{ \% Difference } \\
\cline { 4 - 9 } & & & Mean & SD & $\begin{array}{c}\text { RSD } \\
(\boldsymbol{\%})\end{array}$ & Mean & SD & $\begin{array}{c}\text { RSD } \\
(\%)\end{array}$ & \\
\hline 1 & ER & 750 & 74900 & 3010 & 4 & 79500 & 7580 & 10 & 6 \\
\hline 2 & IR & 850 & 48800 & 4770 & 10 & 62000 & 5060 & 8 & 21 \\
\hline 3 & IR & 1000 & 89300 & 4750 & 5 & 89900 & 12500 & 14 & 1 \\
\hline 4 & IR & 850 & 131000 & 3270 & 2 & 153495 & 9100 & 6 & 14 \\
\hline 5 & IR & 1000 & 9850 & 691 & 7 & 13814 & 851 & 6 & 29 \\
\hline
\end{tabular}




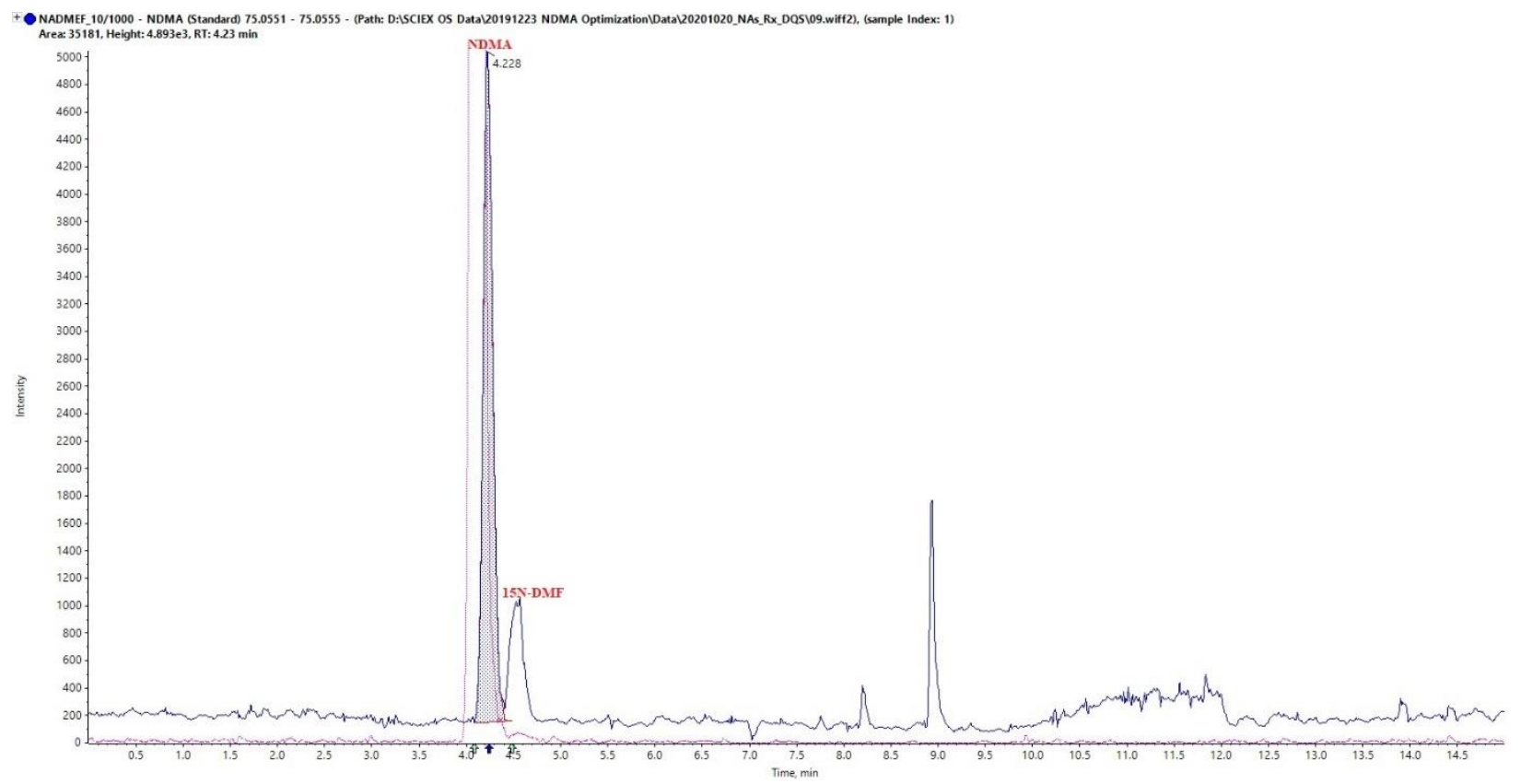

Figure 1. LC separation of NDMA (75.0553) and ${ }^{15} \mathrm{~N}-\mathrm{DMF}$ (75.0571) in the present study. The peak of ${ }^{15} \mathrm{~N}$-DMF shown in this figure is the result of monitoring TOF MS/MS at 75.0553 with the default resolution and mass accuracy of $5 \mathrm{ppm}$.

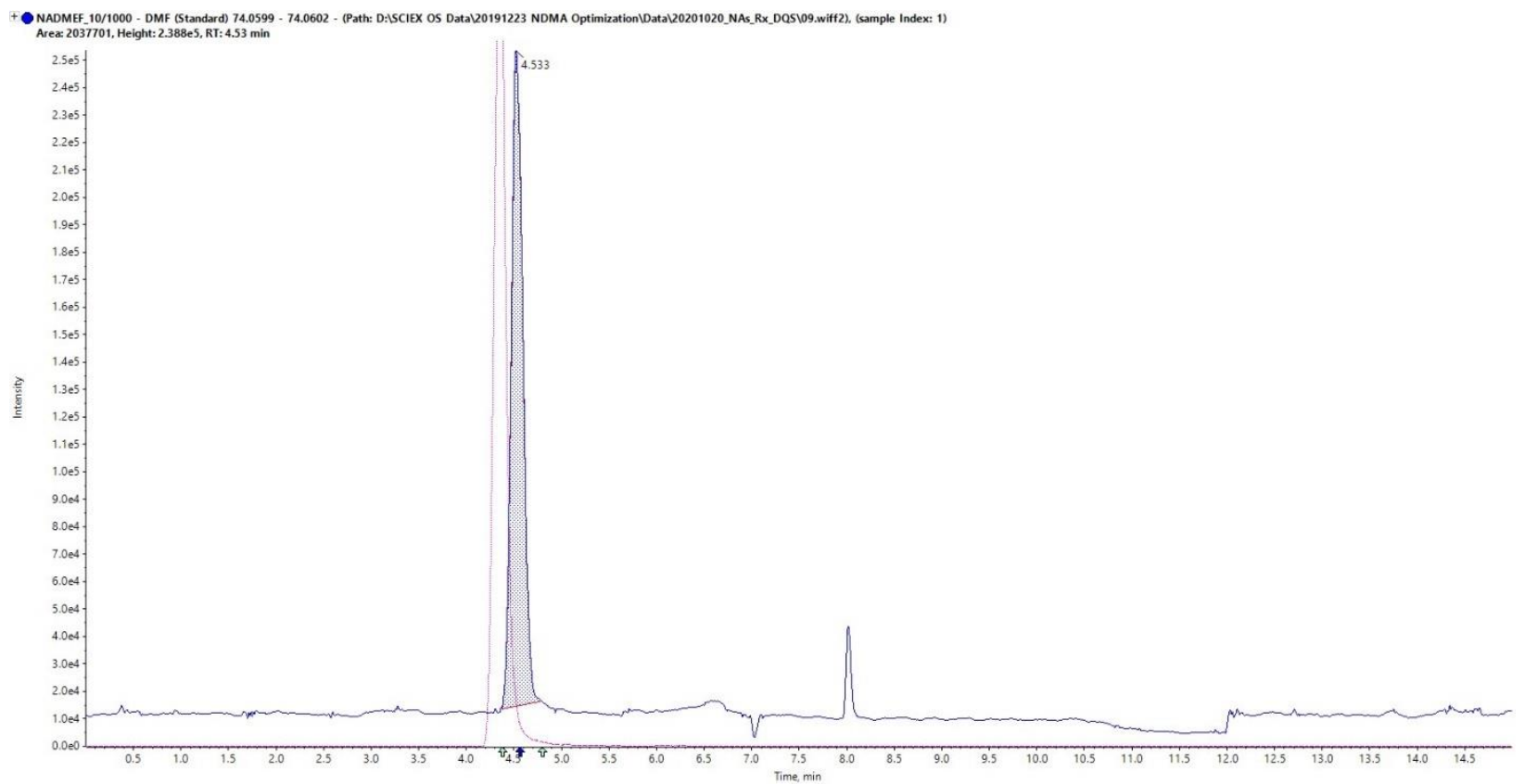

Figure 2. Detection of native DMF (74.0600) at concentration of $1000 \mathrm{ng} / \mathrm{mL}$. 


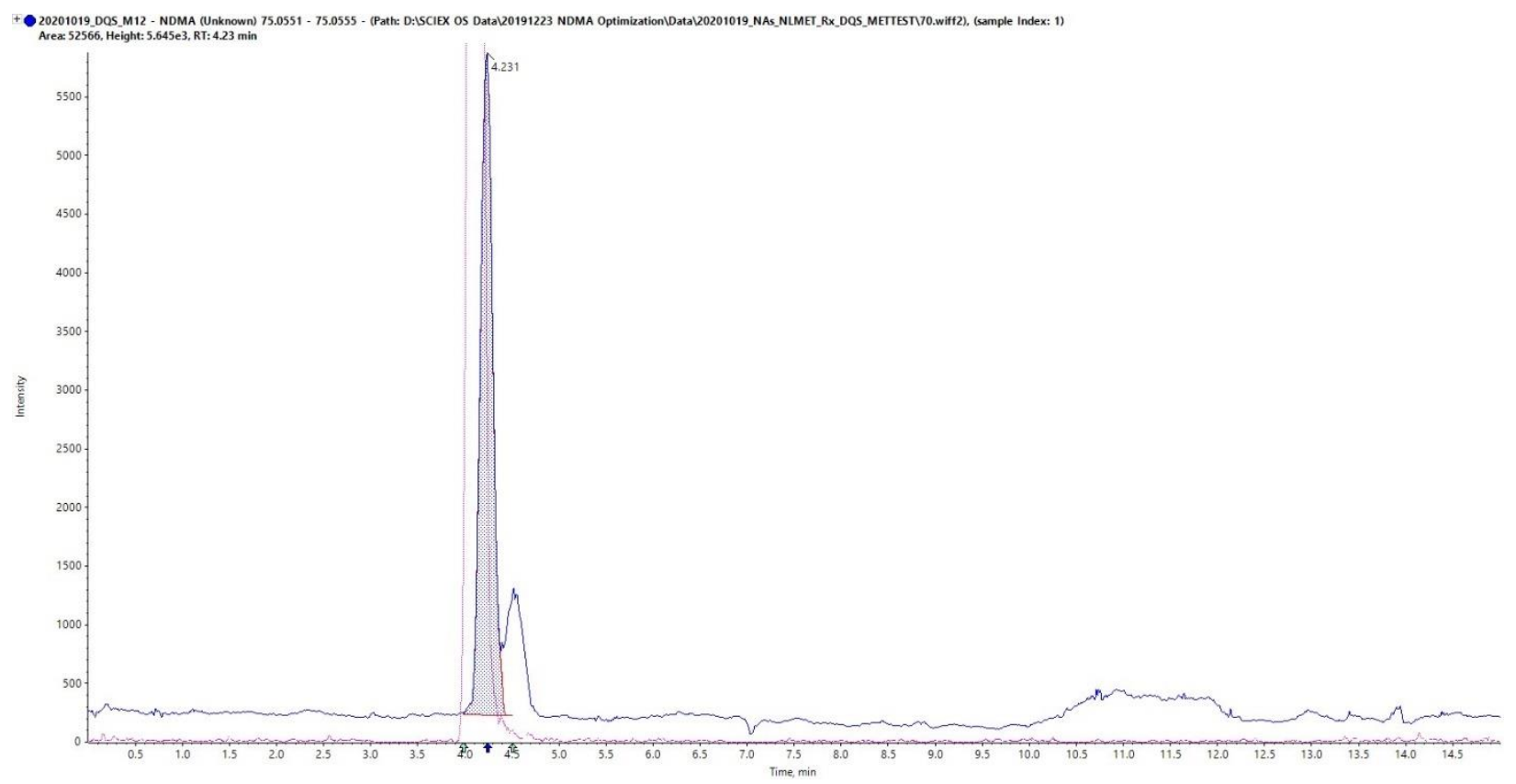

Figure 3. NDMA detection in a real metformin drug sample and DMF is chromatographically separated.
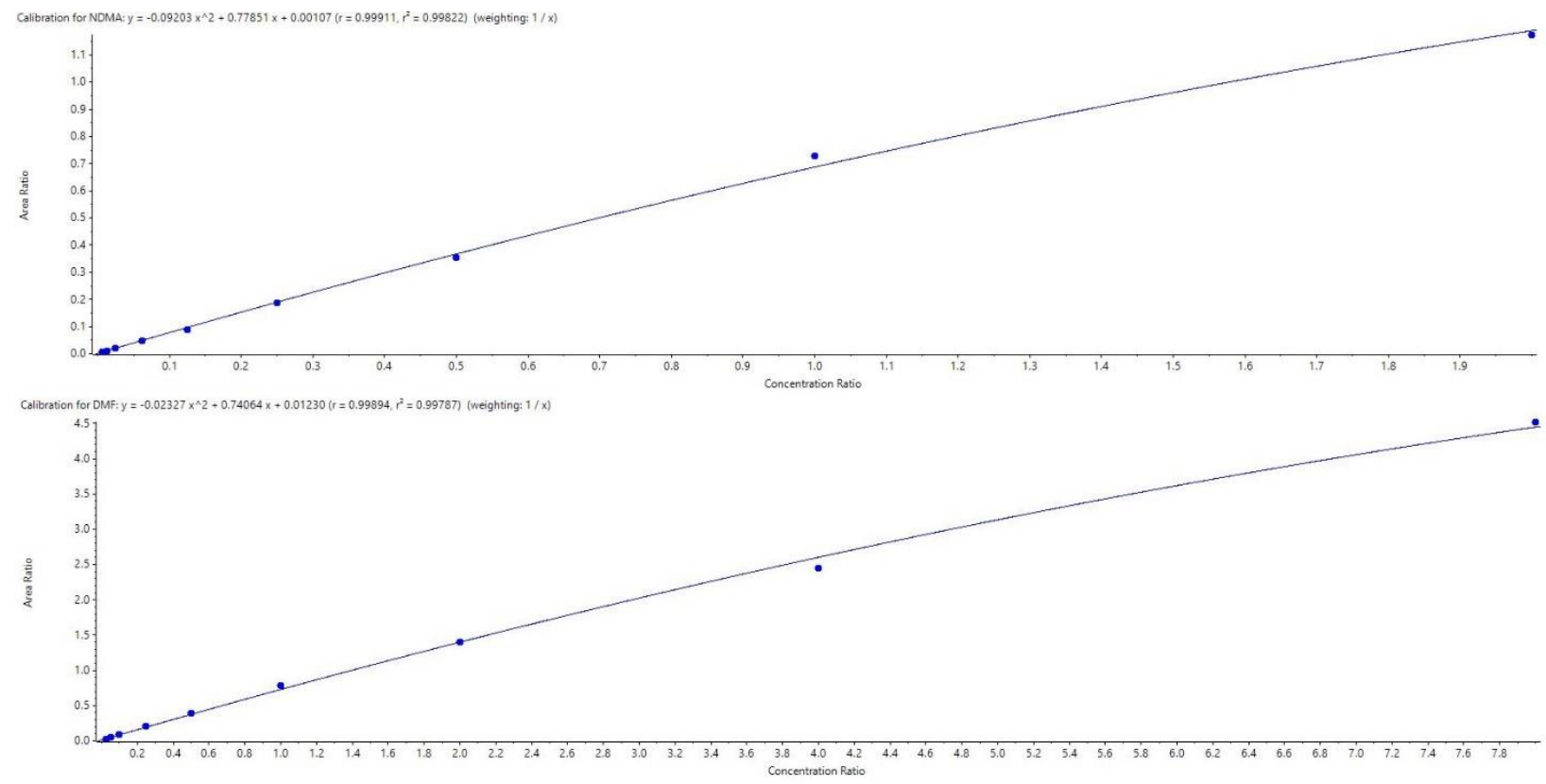

Figure 4. Calibration curves of NDMA and DMF generated with quadratic regression and $1 / \mathrm{x}$ weighting. 